\title{
Quantum spin dynamics in external classical fields
}

\author{
Yuri N. Obukhov ${ }^{1, *}$, Alexander J. Silenko ${ }^{2,3, * *}$, and Oleg V. Teryaev ${ }^{3,4, * * *}$ \\ ${ }^{1}$ Theor. Physics Lab., IBRAE, Russian Academy of Sciences, B. Tulskaya 52, 115191 Moscow, Russia \\ ${ }^{2}$ Research Institute for Nuclear Problems, Belarusian State University, Minsk 220030, Belarus \\ ${ }^{3}$ Bogoliubov Laboratory of Theoretical Physics, Joint Institute for Nuclear Research, Dubna 141980, \\ Russia \\ ${ }^{4}$ National Research Nuclear University "MEPhI”, Kashirskoe Shosse 31, 115409 Moscow, Russia
}

\begin{abstract}
We study the quantum and classical dynamics of spinning particles in the framework of the general-relativistic covariant Dirac theory. The exact Foldy-Wouthuysen transformation for the most general case of a fermion in arbitrary configurations of the gravitational, inertial and electromagnetic fields is derived. We demonstrate the complete consistency of the quantum and classical dynamics. As physical applications, we discuss prospects of probing spacetime structure and using spin effects for gravitational wave detection.
\end{abstract}

\section{Introduction}

Understanding the dynamics of particles with spin (electrons, protons, neutrons, neutrinos) in arbitrary electromagnetic, gravitational and inertial fields is important for many high-energy and astrophysical problems. Moreover, in the consistent gauge-theoretic approach [1], spin of matter is coupled to the spacetime torsion, and hence by measuring spin effects one can probe the spacetime geometry, detecting a possibly nontrivial post-Riemannian geometric structure.

\section{Electromagnetism and gravity in gauge-theoretic approach}

The gauge-theoretic formalism underlies the modern physics, and it is convenient to work in this framework when discussing the dynamics of particles with microstructure (spin and dipole moments) in external classical fields. Fermion matter is a source for the gauge fields which act as mediators of physical interactions. Here we confine our attention only to electromagnetic and gravitational interactions. In the general Yang-Mills-Utiyama-Kibble approach [1], electromagnetism is based on the 1-parameter $U(1)$ symmetry group with the potential 1-form $A=A_{i} d x^{i}$ as the corresponding gauge field, whereas gravity is based on the the 10 parameter Poincaré symmetry group $G=T_{4} \rtimes S O(1,3)$. The gravitational gauge potentials are naturally identified with the coframe $\vartheta^{\alpha}=e_{i}^{\alpha} d x^{i}$ (4 potentials for the translation subgroup $T_{4}$ ) and the local connection $\Gamma^{\alpha \beta}=-\Gamma^{\beta \alpha}=\Gamma_{i}^{\alpha \beta} d x^{i}$ (6 potentials for the Lorentz subgroup

\footnotetext{
*e-mail: obukhov@ibrae.ac.ru

**e-mail: alsilenko@mail.ru

***e-mail: teryaev@theor.jinr.ru
} 
$S O(1,3))$. For the multiplet of gauge potentials,

$$
\left\{\begin{array}{c}
A_{i} \\
e_{i}^{\alpha} \\
\Gamma_{i}^{\alpha \beta}
\end{array}\right\}
$$

we have the corresponding multiplet of the electromagnetic and gravitational ("translational" and "rotational") gauge field strengths:

$$
\left\{\begin{array}{c}
F_{i j}=\partial_{i} A_{j}-\partial_{j} A_{i} \\
T_{i j}{ }^{\alpha}=\partial_{i} e_{j}^{\alpha}-\partial_{j} e_{i}^{\alpha}+\Gamma_{i \beta}{ }^{\beta} e_{j}^{\beta}-\Gamma_{j \beta}{ }^{\alpha} e_{i}^{\beta} \\
R_{i j}^{\alpha \beta}=\partial_{i} \Gamma_{j}^{\alpha \beta}-\partial_{j} \Gamma_{i}^{\alpha \beta}+\Gamma_{i \gamma}{ }^{\beta} \Gamma_{j}^{\alpha \gamma}-\Gamma_{j \gamma}{ }^{\beta} \Gamma_{i}^{\alpha \gamma}
\end{array}\right\} .
$$

For the sake of generality, we assume that the Maxwell tensor $F_{i j}$, the spacetime torsion tensor $T_{i j}{ }^{\alpha}$ and the spacetime curvature tensor $R_{i j}{ }^{\alpha \beta}$ are all nontrivial.

The resulting Riemann-Cartan geometry reduces to the Riemannian spacetime when the torsion vanishes $T_{i j}{ }^{\alpha}=0$. In general, one can decompose the connection into the sum

$$
\Gamma_{i \alpha \beta}=\widetilde{\Gamma}_{i \alpha \beta}-K_{i \alpha \beta}
$$

of the Riemannian connection (denoted by the tilde, it satisfies $\partial_{i} e_{j}^{\alpha}-\partial_{j} e_{i}^{\alpha}+\widetilde{\Gamma}_{i \beta}^{\alpha} e_{j}^{\beta}-\widetilde{\Gamma}_{j \beta}^{\alpha} e_{i}^{\beta}=$ 0 ), and the post-Riemannian contortion tensor which is determined by the torsion,

$$
K_{i \alpha \beta}=\frac{1}{2}\left(T_{\alpha \beta i}-T_{i \alpha \beta}+T_{i \beta \alpha}\right)
$$

Let $x^{i}=\left(t, x^{a}\right)$ be the local coordinates on the spacetime manifold. To study the dynamics of a spinning particle in an arbitrary external fields, one should choose a convenient parametrization of the electromagnetic and gravitational variables. It is common to describe $A_{i}=(-\Phi, \boldsymbol{A})$ in terms of the scalar and vector potentials, whereas the coframe $e_{i}^{\alpha}$ (gravitational translational field) in the Schwinger gauge is parameterized as

$$
e_{i}^{\widehat{0}}=V \delta_{i}^{0}, \quad e_{i}^{\widehat{a}}=W_{b}^{\widehat{a}}\left(\delta_{i}^{b}-c K^{b} \delta_{i}^{0}\right), \quad a=1,2,3 .
$$

We assume that the functions $\Phi, \boldsymbol{A}, V$ and $\boldsymbol{K}$, as well as the components of the $3 \times 3$ matrix $W^{\widehat{a}}{ }_{b}$ may depend arbitrarily on $t, x^{a}$.

\section{Quantum and classical spin dynamics}

In accordance with the equivalence principle (EP), which is a cornerstone of General Relativity theory, gravitational and inertial effects are locally coinciding for classical systems, and motion of small bodies under the action of gravity does not depend on the mass. More recently, the validity of EP for nonrelativistic quantum systems was demonstrated in the experiments of Colella-Overhauser-Werner [2] and Bonse-Wroblewski [3], who measured the phase shift of the wave function of a neutron affected by gravity and inertia, respectively. A natural question arises whether EP can be extended to the relativistic quantum systems, which requires the (comparative) analysis of the quantum and classical spin dynamics in external gravitational and inertial fields [4-6]. 


\subsection{Dirac particle}

We discuss the quantum spin in the framework of the relativistic Dirac theory. The dynamics of a fermion particle with mass $m$ and electric charge $e$ is derived from the Lagrangian

$$
L=\frac{i \hbar}{2}\left(\bar{\psi} \gamma^{\alpha} D_{\alpha} \psi-D_{\alpha} \bar{\psi} \gamma^{\alpha} \psi\right)-m c \bar{\psi} \psi+\frac{1}{2 c} M_{\alpha \beta} \bar{\psi} \sigma^{\alpha \beta} \psi+\frac{i \hbar v^{\prime}}{12} \check{T}^{\alpha} \bar{\psi} \gamma_{\alpha} \gamma_{5} \psi
$$

The first two terms describe a spinor field with the minimal coupling to electromagnetism and gravity which is encoded in the covariant spinor derivative

$$
D_{\alpha}=e_{\alpha}^{i}\left(\partial_{i}-\frac{i e}{\hbar} A_{i}+\frac{i}{4} \Gamma_{i}^{\beta \gamma} \sigma_{\beta \gamma}\right)
$$

In addition, we assume possible nonminimal couplings which are described by the two Paulitype terms in (6), where $M_{\alpha \beta}=\mu^{\prime} F_{\alpha \beta}+c \delta^{\prime} \frac{1}{2} \eta_{\alpha \beta \mu \nu} F^{\mu v}$ is the generalized polarization tensor, and $\check{T}^{\alpha}=-\frac{1}{2} \eta^{\alpha \mu \nu \beta} T_{\mu \nu \beta}=\left\{\check{T}^{0}, \check{T}\right\}$ is the axial torsion. The parameters $\delta^{\prime}, \mu^{\prime}, v^{\prime}$ characterize the magnitude of the corresponding nonminimal couplings.

The components $M_{\hat{0} \hat{a}}=c \mathcal{P}_{a}, M_{\hat{a} \hat{b}}=\epsilon_{a b c} \mathcal{M}^{c}$ are expressed in terms of the 3-vectors

$$
\mathcal{M}=\mu^{\prime} \mathfrak{B}+\delta^{\prime} \mathfrak{E}, \quad \mathcal{P}=c \delta^{\prime} \mathfrak{B}-\mu^{\prime} \mathfrak{E} / c
$$

constructed from the anholonomic magnetic and electric fields which in the coframe (5) read

$$
\mathfrak{E}_{a}=\frac{1}{V} W^{b}(\boldsymbol{E}+c \boldsymbol{K} \times \boldsymbol{B})_{b}, \quad \boldsymbol{B}^{a}=\frac{1}{\operatorname{det} W} W_{b}^{\widehat{a}} \boldsymbol{B}^{b},
$$

where $\boldsymbol{E}=-\nabla \Phi-\frac{\partial \boldsymbol{A}}{\partial t}$ and $\boldsymbol{B}=\nabla \times \boldsymbol{A}$.

Making use of the gravitational field parametrization (5), the Dirac equation derived from (6) can be recast into the Schrödinger form $i \hbar \frac{\partial \psi}{\partial t}=\mathcal{H} \psi$ with the Hermitian Hamiltonian

$$
\begin{aligned}
\mathcal{H}= & \beta m c^{2} V+e \Phi+\frac{c}{2}\left(\pi_{b} \mathcal{F}^{b}{ }_{a} \alpha^{a}+\alpha^{a} \mathcal{F}^{b}{ }_{a} \pi_{b}\right)+\frac{c}{2}(\boldsymbol{K} \cdot \boldsymbol{\pi}+\boldsymbol{\pi} \cdot \boldsymbol{K}) \\
& +\frac{\hbar c}{4}\left(\boldsymbol{\Xi} \cdot \boldsymbol{\Sigma}-\Upsilon \gamma_{5}\right)-\beta V(\boldsymbol{\Sigma} \cdot \boldsymbol{M}+i \boldsymbol{\alpha} \cdot \boldsymbol{P}) .
\end{aligned}
$$

Here: $\boldsymbol{\alpha}=\beta \gamma ; \boldsymbol{\Sigma}$ are the spin matrices; $\boldsymbol{\pi}=-i \hbar \boldsymbol{\nabla}-\boldsymbol{e \boldsymbol { A }}$ is the kinetic momentum operator, and the gravitational (both, GR and post-Riemannian) effects are encoded in

$$
\mathcal{F}^{b}{ }_{a}=V W^{b}, \quad \Upsilon=V \epsilon^{\widehat{a} \widehat{a b c} \widetilde{\Gamma}_{\widehat{a b c}}}+\frac{V c\left(3-v^{\prime}\right)}{3} \check{T}^{0}, \quad \Xi^{a}=\frac{V}{c} \epsilon^{\widehat{a b c} \widetilde{\Gamma}_{\widehat{0 b c}}}-\frac{V\left(3-v^{\prime}\right)}{3} \check{T}^{a} .
$$

To reveal the physical contents of the theory, we use the method developed in Refs. [79] to construct the unitary transformation from the Schrödinger picture (10) to the FoldyWouthuysen (FW) representation. The resulting FW Hamiltonian reads:

$$
\mathcal{H}_{F W}=\beta \epsilon^{\prime}+e \Phi+\frac{c}{2}(\boldsymbol{K} \cdot \boldsymbol{\pi}+\boldsymbol{\pi} \cdot \boldsymbol{K})+\frac{\hbar}{2} \boldsymbol{\Pi} \cdot \boldsymbol{\Omega}_{(1)}+\frac{\hbar}{2} \boldsymbol{\Sigma} \cdot \boldsymbol{\Omega}_{(2)} .
$$

Here $\boldsymbol{\Pi}=\beta \boldsymbol{\Sigma}$ is the polarization operator, and we introduced the 3-vector operators

$$
\begin{aligned}
\Omega_{(1)}^{a}= & \frac{m c^{4}}{2}\left\{\frac{1}{\mathcal{T}},\left\{\pi_{e}, \epsilon^{a b c} \mathcal{F}^{e}{ }_{b} \mathcal{F}^{d}{ }_{c} \partial_{d} V\right\}\right\}+\frac{c^{2}}{8}\left\{\frac{1}{\epsilon^{\prime}},\left\{\pi_{e},\left(2 \epsilon^{a b c} \mathcal{F}^{d}{ }_{b} \partial_{d} \mathcal{F}^{e}{ }_{c}\right.\right.\right. \\
& \left.\left.\left.+\delta^{a b} \mathcal{F}^{e}{ }_{b} \Upsilon\right)\right\}\right\}+\frac{e c^{2}}{4} \epsilon^{a b c}\left\{\frac{1}{\mathcal{T}},\left(\left\{\mathcal{F}^{d}{ }_{b}, \pi_{d}\right\} V^{2} \mathfrak{E}_{c}-V^{2} \mathfrak{E}_{b}\left\{\mathcal{F}^{d}{ }_{c}, \pi_{d}\right\}\right)\right\} \\
& -\frac{c}{4 \hbar} \epsilon^{a b c}\left\{\frac{1}{\epsilon^{\prime}},\left(\left\{\mathcal{F}^{d}{ }_{b}, \pi_{d}\right\} V \mathcal{P}_{c}-V \mathcal{P}_{b}\left\{\mathcal{F}^{d}{ }_{c}, \pi_{d}\right\}\right)\right\}
\end{aligned}
$$




$$
\begin{aligned}
\Omega_{(2)}^{a}= & \frac{c^{2}}{8}\left\{\frac{1}{\mathcal{T}},\left\{\left\{\pi_{e}, \mathcal{F}^{e}{ }_{b}\right\},\left\{\pi_{f},\left[\epsilon^{a b c}\left(\frac{1}{c} \dot{\mathcal{F}}^{f}{ }_{c}-\mathcal{F}^{d}{ }_{c} \partial_{d} K^{f}+K^{d} \partial_{d} \mathcal{F}^{f}{ }_{c}\right)\right.\right.\right.\right. \\
& \left.\left.\left.\left.-\frac{1}{2} \mathcal{F}^{f}{ }_{d}\left(\delta^{d b} \Xi^{a}-\delta^{d a} \Xi^{b}\right)\right]\right\}\right\}\right\}+\frac{c}{2} \Xi^{a}-\frac{e c^{2}}{2}\left\{\frac{1}{\epsilon^{\prime}}, V^{2} \mathfrak{B}^{a}\right\} \\
& -\frac{2 V}{\hbar} \mathcal{M}^{a}+\frac{c^{2}}{2 \hbar}\left\{\frac{1}{\mathcal{T}},\left\{\left\{\delta^{a b} \mathcal{F}^{d}{ }_{b} \mathcal{F}^{e}{ }_{c} V \mathcal{M}^{c}, \pi_{d}\right\}, \pi_{e}\right\}\right\},
\end{aligned}
$$

where the curly brackets $\{$, $\}$ denote anticommutators, and

$$
\epsilon^{\prime}=\sqrt{m^{2} c^{4} V^{2}+\frac{c^{2}}{4} \delta^{a c}\left\{\pi_{b}, \mathcal{F}_{a}^{b}\right\}\left\{\pi_{d}, \mathcal{F}_{c}\right\}}, \quad \mathcal{T}=2 \epsilon^{\prime 2}+\left\{\epsilon^{\prime}, m c^{2} V\right\}
$$

It is important to emphasize that is an exact result in all terms which are of the zero and the first orders in the Planck constant $\hbar$, and we did not make any assumptions about the weakness of the external fields. In other words, our findings are applicable to any configurations of external fields which can be arbitrarily strong. Although the final formulas look similar to those derived earlier in [10], one should notice that now (11) include the contributions from the nontrivial torsion field.

\subsection{Classical particle with spin}

Classical theory of spin was developed quite early after the concept of spin was introduced for elementary particles [11-15]. This theory underlies the analysis of dynamics of polarized particles in accelerators and storage rings.

The motion of a test particle is described by its 4-velocity $u^{\alpha}$ and by the 4-vector of spin $S^{\alpha}$ which satisfy the normalization, $u_{\alpha} u^{\alpha}=c^{2}$, and the orthogonality, $S_{\alpha} u^{\alpha}=0$, conditions. By neglecting the second order spin effects $[16,17]$, the dynamical equations for the spinning particle in external electromagnetic and gravitational fields read

$$
\begin{aligned}
\frac{D u^{\alpha}}{d \tau} & =-\frac{e}{m} F_{\beta}^{\alpha} u^{\beta}, \\
\frac{D S^{\alpha}}{d \tau} & =-\left(v^{\prime}-2\right) u^{i} K_{i \beta}{ }^{\alpha} S^{\beta}-\frac{e}{m} F_{\beta}^{\alpha} S^{\beta}-\frac{2}{\hbar}\left[M^{\alpha}{ }_{\beta}+\frac{u^{\gamma}}{c^{2}}\left(u^{\alpha} M_{\beta \gamma}-u_{\beta} M_{\gamma}^{\alpha}\right)\right] S^{\beta} .
\end{aligned}
$$

The laboratory-frame components of the vector $S^{\alpha}$ do not describe the physical spin of a particle: We have to recall that spin, as the "internal angular momentum" of a particle, is determined with respect to the rest reference frame.

We denote the physical spin by $\boldsymbol{s}$. Using the general scheme developed in $[10,18,19]$, we reduce the system (16)-(17) to

$$
\frac{d \boldsymbol{s}}{d \tau}=\mathbf{\Omega} \times \boldsymbol{s}
$$

Notice that $u^{\alpha}=\{\gamma, \gamma \boldsymbol{v}\}$ with the Lorentz factor $\gamma$. We find the precession angular velocity

$$
\boldsymbol{\Omega}=\frac{e}{m}\left(-\boldsymbol{B}_{\mathrm{eff}}+\frac{\gamma}{\gamma+1} \frac{\boldsymbol{v} \times \mathfrak{E}_{\mathrm{eff}}}{c^{2}}\right)
$$

as a function of the external fields which enter via the effective fields:

$$
\begin{aligned}
\mathfrak{E}_{\mathrm{eff}} & =\mathfrak{E}-\frac{2 m}{e \hbar} \gamma^{2} \boldsymbol{v} \times \boldsymbol{\Delta}+\frac{m}{e} \mathcal{E}, \\
\mathfrak{B}_{\mathrm{eff}} & =\mathfrak{B}+\frac{2 m}{e \hbar} \gamma^{2}\left[\boldsymbol{\Delta}-\frac{1}{c^{2}} \boldsymbol{v}(\boldsymbol{v} \cdot \boldsymbol{\Delta})\right]+\frac{m}{e} \mathcal{B} .
\end{aligned}
$$


Here the polarization current combines the effects of nonminimal couplings

$$
\Delta=\mathcal{M}+\frac{1}{c} v \times \mathcal{P}
$$

whereas the gravitoelectric and gravitomagnetic fields

$$
\mathcal{E}=\widetilde{\mathcal{E}}-\frac{\gamma c\left(3-v^{\prime}\right)}{6} \boldsymbol{v} \times \check{\boldsymbol{T}}, \quad \mathcal{B}=\widetilde{\mathcal{B}}+\frac{\gamma c\left(3-v^{\prime}\right)}{6}\left(\check{\boldsymbol{T}}-\boldsymbol{v} \check{T}^{0}\right)
$$

encompass GR terms $\widetilde{\mathcal{E}}, \widetilde{\mathcal{B}}$ and the torsion contributions.

\subsection{Consistency of quantum and classical spin dynamics}

The quantum spin dynamics is determined from the commutator of the polarization operator $\boldsymbol{\Pi}=\beta \boldsymbol{\Sigma}$ with the FW Hamiltonian (12):

$$
\frac{d \boldsymbol{\Pi}}{d t}=\frac{i}{\hbar}\left[\mathcal{H}_{F W}, \boldsymbol{\Pi}\right]=\mathbf{\Omega}_{(1)} \times \mathbf{\Sigma}+\mathbf{\Omega}_{(2)} \times \mathbf{\Pi} .
$$

To derive the corresponding semiclassical expressions from the quantum results, we should evaluate all anticommutators and neglecting the powers of $\hbar$ higher than 1 in (13)-(15). Then (24) reduces to (18), and we can verify that $\boldsymbol{\Omega}=\boldsymbol{\Omega}_{(1)}+\boldsymbol{\Omega}_{(2)}$ is exactly equal to (19) after we notice the identifications [10]

$$
\beta \frac{c^{2}}{\epsilon^{\prime}} \mathcal{F}^{b}{ }_{a} \pi_{b}=v_{a}, \quad \frac{\epsilon^{\prime}}{\epsilon^{\prime}+m c^{2} V}=\frac{\gamma}{1+\gamma}, \quad \frac{c^{3}}{\epsilon^{\prime}\left(\epsilon^{\prime}+m c^{2} V\right)} \mathcal{F}^{b}{ }_{a} \pi_{b} \mathcal{F}^{d}{ }_{c} \pi_{d}=\frac{\gamma}{1+\gamma} \frac{v_{a} v_{c}}{c} .
$$

This general result is of fundamental importance for the comparison of the dynamics of a quantum and classical spinning particle in external fields for the proof of the validity of the equivalence principle, extended to relativistic quantum systems.

\section{Physical effects: probing spacetime structure}

Making use of the theoretical framework outlined here, one can establish observational bounds on spin-torsion coupling from the dynamics of freely precessing nuclear spins in a uniform magnetic field $\boldsymbol{B}$. From the data [20] of the experiment with ${ }^{199} \mathrm{Hg}$ and ${ }^{201} \mathrm{Hg}$ atoms devoted to the search of a hypothetical scalar-pseudoscalar interactions, we derive [18] the strong restriction on the absolute value of the spacetime torsion:

$$
\frac{\hbar c}{4}|\check{\boldsymbol{T}}| \cdot|\cos \Theta|<2.2 \times 10^{-21} \mathrm{eV}, \quad|\check{\boldsymbol{T}}| \cdot|\cos \Theta|<4.3 \times 10^{-14} \mathrm{~m}^{-1}
$$

Here $\Theta$ is the angle between $\boldsymbol{B}$ and the torsion $\check{\boldsymbol{T}}$. One can further improve these bounds by approximately one order by making use of the more recent experimental data [21].

As another application, one could develop a novel technique to probe gravitational waves by observing the spin dynamics in a magnetic resonance setup [10]. In the local coordinates $(t, x, y, z)$, the spacetime geometry of a weak gravitational wave is given by

$$
V=1, \quad K=0, \quad W_{b}^{\widehat{a}}=\left(\begin{array}{ccc}
1+w_{\oplus} & w_{\otimes} & 0 \\
w_{\otimes} & 1-w_{\oplus} & 0 \\
0 & 0 & 1
\end{array}\right)
$$


The two independent polarizations of a wave with the frequency $\omega$ propagating along the $z$ axis are described by the functions $w_{\otimes}=w_{\otimes}(\varphi)$ and $w_{\oplus}=w_{\oplus}(\varphi)$ of the phase $\varphi=$ $\omega(t-z / c)$. Suppose $w_{\oplus}=0$ and $w_{\otimes}=g_{0} \cos \varphi$, and we apply a constant homogeneous magnetic field in the plane of the wave front: $\boldsymbol{B}=\left(B_{0}, 0,0\right)$, with $B_{0}=$ const. Then from (9) we find the field acting on the spin: $\mathfrak{B}=\left(B_{0}, B_{0} w_{\bigotimes}, 0\right)$. Thereby, the resulting field configuration reproduces the magnetic resonance conditions, when the spin is affected by the constant homogeneous magnetic field along $x$ superimposed with an additional alternating field in the perpendicular plane $(y, z)$. Computing the probability of the spin-flip we find that the effect is quadratic in the wave amplitude $g_{0}$. However, one can expect that also the polarization effects which are linear in $g_{0}$ can possibly show up in the analysis of the spin components orthogonal to initial spin polarization.

\section{Conclusion}

Our results on the quantum and quasiclassical equations of motion of spinning particles are of direct operational relevance and they form the basis for many experiments in high-energy physics and astrophysics. Important applications range from the study of polarized beams in accelerators and storage rings to the development of novel methods of gravitational wave detection.

The experimental study of the action of a gravitational wave on spin might provide a useful supplementary technical tool for the existing modern experiments (LIGO/VIRGO). In particular, one could search for a very weak (possibly amplified by means of an applied magnetic field) effect of the gravitational wave on spin in coincidence with the standard signal.

Acknowledgments. The work was supported in part by the Belorussian Republican Foundation for Fundamental Research (Grant Ф18D-002), by the Heisenberg-Landau program of the German Ministry for Science and Technology (Bundesministerium für Bildung und Forschung), and by the Russian Foundation for Basic Research (Grants 18-02-01107 and 16-02-00844-A).

\section{References}

[1] M. Blagojević and F. W. Hehl, eds, Gauge Theories of Gravitation. A Reader with Commentaries (Imperial College Press: London, 2013) 635

[2] R. Colella, A.W. Overhauser, and S.A.Werner, Phys. Rev. Lett. 34, 1472 (1975)

[3] U. Bonse and T. Wroblewski, Phys. Rev. Lett. 51, 1401 (1983)

[4] O.V. Teryaev, Spin structure of nucleon and equivalence principle, arXiv: hep$\mathrm{ph} / 9904376$ (1999)

[5] Yu.N. Obukhov, Phys. Rev. Lett. 86, 192 (2001)

[6] A.J. Silenko and O.V. Teryaev, Phys. Rev. D 71, 064016 (2005)

[7] A.J. Silenko, Phys. Rev. A 77, 012116 (2008)

[8] A.J. Silenko, Phys. Rev. A 91, 012111 (2015)

[9] A.J. Silenko, Phys. Rev. A 91, 022103 (2015)

[10] Yu.N. Obukhov, A.J. Silenko, and O.V. Teryaev, Phys. Rev. D 96, 105005 (2017)

[11] J. Frenkel, Zeits. Phys. 37, 243 (1926)

[12] L.H. Thomas, Phil. Mag. 3, 1 (1927)

[13] M. Mathisson, Acta Phys. Pol. 6, 163 (1937)

[14] V. Bargmann, L. Michel, and V.L. Telegdi, Phys. Rev. Lett. 2, 435 (1959)

[15] H.C. Corben, Classical and quantum theories of spinning particles (Holden-Day, Inc: San Francisco, 1968) 279 
[16] C. Chicone, B. Mashhoon, and B. Punsly, Phys. Lett. A 343, 1 (2005)

[17] B. Mashhoon and D. Singh, Phys. Rev. D 74, 124006 (2006)

[18] Yu.N. Obukhov, A.J. Silenko, and O.V. Teryaev, Phys. Rev. D 90, 124068 (2014)

[19] Yu.N. Obukhov, A.J. Silenko, and O.V. Teryaev, Phys. Rev. D 94, 044019 (2016)

[20] B.J. Venema, P.K. Majumder, S.K. Lamoreaux, B.R. Heckel, and E.N. Fortson, Phys. Rev. Lett. 68, 135 (1992)

[21] C. Gemmel et al., Eur. Phys. J. D 57, 303 (2010) 